\title{
Orientación a Objetivos de Logro en Estudiantes de Educación Superior
}

\section{Achievement Goal Oriented Approach in Higher Education Students}

\author{
Aguilar Jacal Rosa Elena*, Gallardo Aguilar María del Consuelo**, \\ De la Garza Carranza María Teresa**
}

\begin{abstract}
*Estudiante de Maestría en Gestión Administrativa. División de Ciencias Económico-Administrativas del Instituto Tecnológico Nacional de México en Celaya. Email: m2003029@itcelaya.edu.mx. ORCID: https://orcid.org/0000-0001-7380-813X.

**Maestra en Administración por la Facultad de Contaduría y Administración de la Universidad Autónoma de México. Profesor Investigador del Instituto Tecnológico Nacional de México en Celaya. Email: consuelo.gallardo@itcelaya.edu.mx. ORCID: https://orcid.org/0000-0003-3591-2379.

${ }^{* *}$ Doctora en Ciencias Administrativas por el Instituto Politécnico Nacional. Profesor Investigador del Instituto Tecnológico Nacional de México en Celaya. Email: teresa.garza@itcelaya.edu.mx. ORCID: https://orcid.org/0000-0002-4877-3403.
\end{abstract}

Correo para recibir correspondencia: m2003029@itcelaya.edu.mx

Fecha de recibido: 19 de abril de 2021

Fecha de aceptación: 27 de julio de 2021 


\section{RESUMEN}

Existen diferentes enfoques de objetivos que adoptan los estudiantes, los cuales pueden ser, enfocarse al rendimiento escolar o adquirir mayor conocimiento.

OBJETIVO: Analizar un instrumento de investigación para saber si es confiable, el cual pretende determinar las orientaciones de los estudiantes en una institución de educación superior, además de analizar que orientación tienen los hombres respecto y las mujeres.

MATERIAL Y MÉTODO: Se realizó la prueba de estudio con alumnos del nivel superior, se obtuvieron 104 respuestas. Las pruebas aplicadas al estudio fueron: análisis factorial, análisis de fiabilidad, prueba $T$ y prueba $F$ de ANOVA.

RESULTADOS: El cuestionario es fiable para poder aplicarse en investigaciones futuras, además que no hay una tendencia marcada de hombres y mujeres en cuanto a la orientación de objetivos.

CONCLUSIONES: Se encontró que no hay diferencia significativa en la orientación de objetivos de hombres respecto de mujeres, cuando en la teoría mencionan que existe una diferencia muy marcada porque los hombres tienden a orientarse hacia objetivos de rendimiento y las mujeres a objetivos de aprendizaje.

PALABRAS CLAVE: Objetivos de aprendizaje. Objetivos de rendimiento. Logro académico.

\section{ABSTRACT}

There are different goal approaches that students adopt which can be, implemented to focus on school performance or to acquire greater knowledge.

OBJECTIVE: To analyze a research instrument to know if it is reliable. The aim is to determine Higher Education students' orientations, and to analyze what orientation men and women have.

MATERIAL AND METHOD: A test was carried out with Higher Education students, obtaining 104 responses. Tests applied to the study: factorial, and reliability analysis, ANOVA T, and Ftests. 
ORIENTACIÓN A OBJETIVOS DE LOGRO EN ESTUDIANTES DE EDUCACIÓN SUPERIOR.

RESULTS: The survey is reliable and can be applied in future research, besides, there is no marked tendency, in men and women, related to goal orientation.

CONCLUSIONS: It was found that there is no significant difference in the goal orientation in men compared to women, when indicated in theory that there is a very marked difference because men tend to be oriented towards performance objectives and women towards learning objectives.

KEY WORDS: Learning objectives. Performance objectives. Academic achievement.

\section{INTRODUCCIÓN}

En el ámbito educativo se entiende por orientación a objetivos de logro a la manera en la que un estudiante entiende las tareas/trabajos y a la forma en la que reacciona ante éstas; es decir, a la forma en como un estudiante logra resolver sus trabajos/tareas, esto deriva en distintos patrones de comportamiento de cada uno de ellos (Dweck \& Leggett, 1988). De acuerdo con Shatz (2015) las orientaciones de los estudiantes a objetivos de logro se asocian altamente con un buen rendimiento académico. Esta teoría plantea que se puede visualizar los objetivos de logro académicos que tienen los estudiantes, tomando en cuenta los propósitos que ellos siguen mientras realizan sus trabajos y tareas académicas (Ames, 1992). Lo cual, se puede realizar con la observación y analizando lo que piensan los alumnos sobre sus tareas y su rendimiento escolar (Ames \& Archer, 1988). Existen dos formas en las que se puede enfocar un alumno en cuanto a sus objetivos de logro académico, la primera es cuando está orientado hacia objetivos de rendimiento y la segunda es cuando se enfoca hacia objetivos de aprendizaje, esto depende de la meta del alumno, la última se refiere a cuando el alumno se enfoca en desarrollar su capacidad de ampliar su conocimiento, y la primera se refiere a que sólo quiera demostrar su capacidad de superioridad frente a los demás alumnos (sus compañeros de clase) y profesores.

\section{Antecedentes}

La motivación en base a objetivos de logro comenzó a estudiarse en 1940, en el trabajo de David McClelland y sus colegas en donde encontraron que existe un vínculo entre el logro y la 
ORIENTACIÓN A OBJETIVOS DE LOGRO EN ESTUDIANTES DE EDUCACIÓN SUPERIOR.

motivación a objetivos. En su estudio, demostraron que se puede predecir el rendimiento académico de los estudiantes tomando en cuenta las orientaciones de objetivos de logro que tienen. Aquellos estudiantes que están orientados al rendimiento académico suelen ser muy competitivos, aspiran a tener éxito y les gustan los desafíos mientras que, por otro lado, los estudiantes sin objetivos determinados tienden a fracasar académicamente y evaden los desafíos en cualquier tarea (Atkinson, 1957). Algunos investigadores de psicología social decidieron ampliar el trabajo realizado por McClelland analizando las representaciones cognitivas y como se van formando las experiencias sociales. Exploraron la motivación de logro como un aspecto de la identidad y en los patrones de pensamiento que se dan en varios contextos (Weiner, 1979).

Como resultado de la investigación, se logró demostrar que la orientación al logro de un individuo puede ser caracterizada por uno de dos perfiles distintos: orientación al aprendizaje y orientación al rendimiento.

\section{Marco Teórico}

\section{Modelo de orientación a objetivos}

Orientación al rendimiento: un individuo orientado al rendimiento es aquel que cree que el éxito resulta de la inteligencia superior y de lograr los mejores resultados en comparación a los demás (Senko, 2002). Las personas que se orientan al rendimiento son aquellos que quieren superar a los que los rodean y demostrarles su capacidad; sin embargo, este tipo de orientación puede llegar a tener efectos negativos como, por ejemplo, evadir las situaciones desafiantes o resultados bajos debido al alto nivel alto estrés y de frustración por sobresalir.

Orientación al aprendizaje: un individuo orientado al aprendizaje es aquel que considera que el éxito resulta del esfuerzo en combinación con el uso de estrategias adecuadas. Las personas orientadas al aprendizaje trabajan en desarrollar su habilidad, comprensión y conocimiento en una tarea determinada. Muchos estudios han demostrado que este tipo de orientación fomenta el aprendizaje de tipo adaptativo a las diferentes situaciones, lo cual hace que un estudiante logre un alto rendimiento académico (Diener \& Dweck, 1978). Por ejemplo, los alumnos que se orientan al aprendizaje tienen una mayor motivación para aprender, usan y desarrollan estrategias de conocimiento y se vuelven mejores a través de desafíos y fracasos (Ames, 1984). 
ORIENTACIÓN A OBJETIVOS DE LOGRO EN ESTUDIANTES DE EDUCACIÓN SUPERIOR.

\section{Modelo de orientación a objetivos de cuatro factores}

Los estudios más recientes en cuanto orientación a logros hicieron un desglose del modelo de los dos factores (orientación al rendimiento y orientación al aprendizaje) agregando los elementos de enfoque y evasión (Elliot \& Harackiewicz, 1996). Como resultado, se obtuvieron cuatro enfoques de logro: de rendimiento, evasión de rendimiento, aprendizaje y evasión de aprendizaje (Ames, 1984).

Enfoque de rendimiento: Esta persona se orienta en demostrar una alta capacidad académica para sí mismo y para los demás (Wolters, 2004).

Enfoque evasión de rendimiento: Esta persona se orienta en parecer menos capaces que las personas que los rodean (Wolters, 2004).

Enfoque al aprendizaje: Esta persona tiene como meta absorber todo el conocimiento que le sea posible y realiza todas las actividades que se le asignan de la mejor manera posible (Wolters, 2004).

Enfoque de evasión de aprendizaje: Esta persona no tiene interés en adquirir nuevos conocimientos, se centran en hacer sus tareas un poco mejor que las anteriores con el mínimo esfuerzo (Wolters, 2004).

\section{Teorías de inteligencia}

De acuerdo con el de trabajo de Mueller \& Dweck (1998) las teorías de la inteligencia se refieren a las creencias que tiene un individuo sobre la capacidad intelectual que tienen otras personas. Es decir, si la inteligencia es innata a un individuo o si es una característica que se puede modificar. La percepción de la inteligencia influye en factores motivacionales los cuales van asociados a la orientación de objetivos de logro, esto puede influir en el rendimiento académico que tiene un alumno (Mueller \& Dweck, 1998), las teorías de la inteligencia son:

Teoría incremental: Este tipo de personas creen que la inteligencia se desarrolla, porque es el resultado del esfuerzo de una persona aplicado a situaciones desafiantes (Blackwell, Trzesniewski \& Dweck, 2007).

Teoría de la entidad: Cuando una persona considera que la inteligencia es fija, cree que es una característica innata de un individuo (Mueller \& Dweck, 1998). En resumen, se trata de si eres 
ORIENTACIÓN A OBJETIVOS DE LOGRO EN ESTUDIANTES DE EDUCACIÓN SUPERIOR.

inteligente o no eres inteligente. Esto tiene efectos negativos en los estudiantes porque los lleva a mentalizarse que el esfuerzo tiene mínima importancia en resultados académicos (Kamins \& Dweck, 1999).

\section{Mentalidad}

Se define como la percepción que tiene una persona sobre sí mismo, sus capacidades, talentos e inteligencia. Estas percepciones o creencias influyen en los aspectos de vida de un individuo como el rendimiento educativo, además en la vida profesional, deportistas y relaciones interpersonales (Hattie \& Timperley, 2007), los tipos de mentalidad son:

Mentalidad fija: Es cuando una persona cree que la inteligencia no se puede cambiar de una manera significativa, como si la inteligencia fuera determinada por genética. Este tipo de personas creen que no existe ninguna relación entre el esfuerzo y éxito (Dweck, 2010).

Mentalidad de crecimiento: Las personas que tienen este tipo de mentalidad creen que la inteligencia, talentos y habilidades de una persona se desarrollan con la práctica. Este tipo de personas creen que tienen el control del éxito que tengan, por lo cual ellos pueden resolver mejor las situaciones problemáticas (Dweck, 2010).

\section{Factores que influyen en la orientación a objetivos}

Ha sido demostrado que, al combinar ciertos factores de tipo motivacionales, cognitivos y de contexto influyen en las orientaciones de objetivos de logro, los cuales son:

Elogios: Existe evidencia que los elogios que recibe una persona son un factor que influye en el desarrollo de orientación de objetivos (Hattie \& Timperley, 2007). Existen diferentes tipos de elogio y el que se reciba no sólo afecta en el comportamiento, emoción, creencia y resultados inmediatos, si no que puede tener resultados de largo plazo. Esto afecta en la forma que un individuo enfrenta las dificultades y/o problemas futuros (Cimpian, Arce \& Markman, 2007). Los elogios de tipo verbal son otorgados con el afán de reforzar y aumentar el desempeño de los alumnos; sin embargo, algunos elogios pueden tener resultados negativos en el receptor, debilitando su desempeño pues a menudo en vez de aumentar la seguridad en el estudiante crea inseguridad en cuanto sus conocimientos, afectando el rendimiento académico de éste (Henderlong, 2002). 
ORIENTACIÓN A OBJETIVOS DE LOGRO EN ESTUDIANTES DE EDUCACIÓN SUPERIOR.

Edad: La edad es muy relevante para predecir la orientación de objetivos de un estudiante. Por lo general los más jóvenes tienden a adoptar una posición de rendimiento (Midgley \& Anderman, 1995). Cuando se encuentran en la educación superior, los estudiantes por lo general demuestran tener una orientación de aprendizaje; por otro lado, se ha observado que los adolescentes en general tienden a mostrar disminución en la motivación de sus estudios (Eccles, et al., 1993).

Género: Muchos trabajos de investigación han obtenido como resultados, que las mujeres tienden orientarse de manera motivacional, la cual algunas veces no ayuda en tener un alto rendimiento académico, especialmente en el área de matemáticas (Dweck, 1986). Por otro lado, los resultados de los trabajos de investigación que se encargan de analizar las diferencias entre género en la orientación a objetivos han tenido resultados muy variados. Por ejemplo, en el trabajo de Carol Dweck (1986) en el cual encontró que las diferencias de género se deben a factores externos, y que las mujeres se orientan a objetivos de rendimiento. Además, de acuerdo con otros trabajos más recientes se ha encontrado que las mujeres en su mayoría se orientan a objetivos de aprendizaje, mientras que los hombres son quienes se orientan a objetivos de rendimiento y siempre se ve la orientación a objetivos muy marcada en ambos géneros (Meece \& Holt, 1993).

\section{OBJETIVO}

Analizar el instrumento planteado de orientación a objetivos de logro, para saber si es confiable para futuras investigaciones centradas en conocer la orientación a objetivos de logro académico que tienen los estudiantes de nivel superior.

\section{MATERIAL Y MÉTODO}

En este apartado, se describen las características de la muestra y los métodos que se empelaron para el estudio aplicado. Se contó con la participación de estudiantes de licenciatura del Instituto Tecnológico Nacional de Celaya. La encuesta se envió a través del correo institucional de los estudiantes. El cuestionario se realizó en la plataforma Google Forms, y la cantidad de sujetos a contemplarse era un mínimo de 30 (que es el número mínimo para realizar análisis estadísticos); sin embargo, se recibieron 104 respuestas. El tipo de investigación que se aplicó fue tipo mixta, porque se quería lograr un mayor entendimiento del fenómeno de estudio y transversal, porque sólo se realizó un levantamiento de información en un momento determinado. 
ORIENTACIÓN A OBJETIVOS DE LOGRO EN ESTUDIANTES DE EDUCACIÓN SUPERIOR.

Se planteó un instrumento de investigación para saber la orientación a objetivos que tienen los estudiantes del Instituto Tecnológico Nacional de Celaya, para realizar dicho instrumento se realizó una adaptación al cuestionario realizado por Button (1996), en la cual se midió la orientación a objetivos (Tabla 1):

\section{Tabla 1}

Orientación a objetivos de rendimiento y aprendizaje

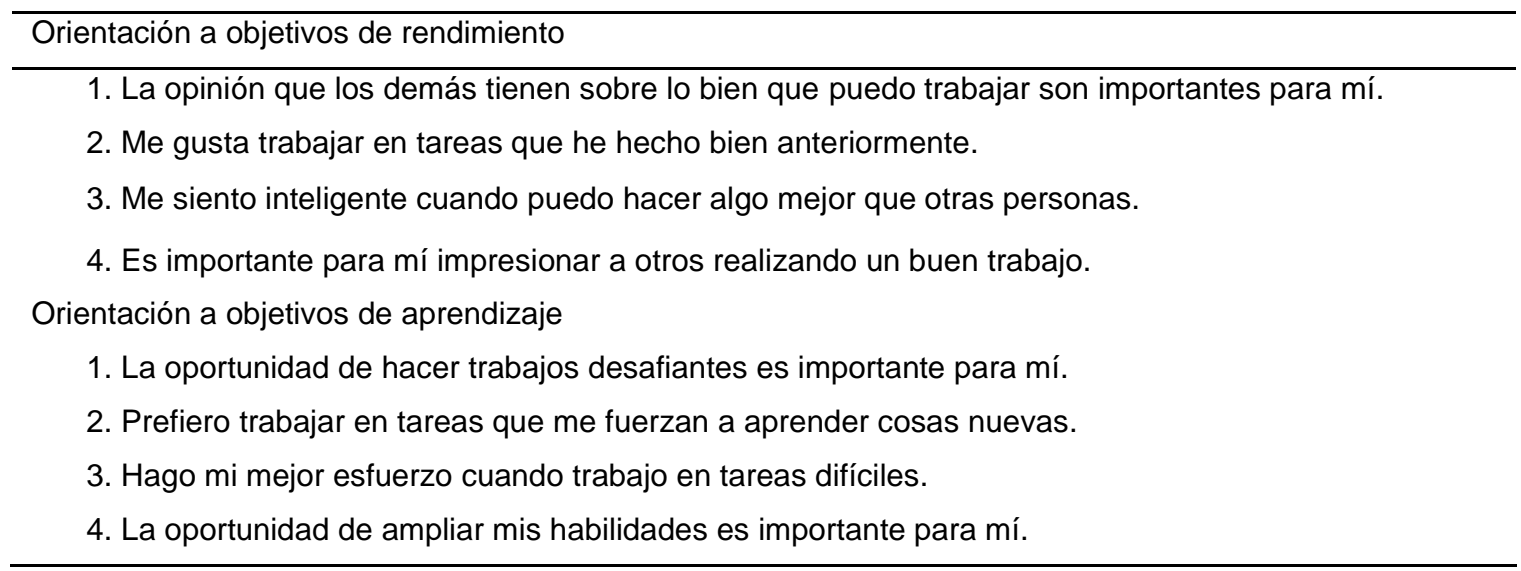

Nota: Son las variables que se utilizaron en el cuestionario, para realizarlo se basó en el trabajo de Button (1966) para medir los objetivos orientados al rendimiento y aprendizaje.

Fuente: Elaboración propia.

\section{RESULTADOS}

Para poder cumplir los objetivos del presente trabajo se realizaron los siguientes análisis:

\section{Análisis factorial}

Este tipo de análisis, es una técnica de reducción de datos que sirve para encontrar grupos homogéneos de variables a partir de un conjunto numeroso de variables. Su propósito consiste en buscar el número mínimo de dimensiones, capaces de explicar el máximo de información contenida en los datos (De la Fuente, 2011).

Tabla 2

Prueba de KMO y Bartlett

\begin{tabular}{lll}
\hline Medida Kaiser-Meyer-Olkin de adecuación de muestreo & .655 \\
\hline Prueba de esfericidad de Aprox. Chi-cuadrado & 166.752 \\
Bartlett & gl & 28 \\
& Sig. & .000 \\
\hline
\end{tabular}

Fuente: Elaboración propia. 
ORIENTACIÓN A OBJETIVOS DE LOGRO EN ESTUDIANTES DE EDUCACIÓN SUPERIOR.

Después de realizar este análisis, (Tabla 2), que la medida KMO tiene un valor de .655, este valor se encuentra dentro del rango aceptable, lo cual quiere decir que el cuestionario tiene las características apropiadas para realizar un análisis factorial.

Tabla 3

Comunalidades

\begin{tabular}{lll}
\hline & Inicial & Extracción \\
\hline V1 & 1.000 & .225 \\
V2 & 1.000 & .448 \\
V3 & 1.000 & .664 \\
V4 & 1.000 & .636 \\
V5 & 1.000 & .605 \\
V6 & 1.000 & .366 \\
V7 & 1.000 & .501 \\
V8 & 1.000 & .603 \\
\hline
\end{tabular}

Fuente: Elaboración propia.

En cuanto al análisis de las comunalidades en la tabla 3, en su mayoría se encuentran aceptables porque están arriba del nivel aceptable 0.4; sin embargo, hay dos variables que están por debajo de ese nivel (con valores de 366 y .225) aunque la que se podría aceptar porque está muy cercana al 0.4 es la V6 con un valor de .366. A partir de esta tabla, se puede empezar a plantear si el número de factores obtenidos es suficiente para explicar todas y cada una de las variables incluidas en el análisis. También ayudará a plantear si se da por bueno el número de factores extraído, alguna de las variables incluidas podría quedar fuera del análisis.

Tabla 4

Varianza total explicada

\begin{tabular}{|c|c|c|c|c|c|c|c|c|c|}
\hline \multirow[b]{2}{*}{ Componente } & \multicolumn{3}{|c|}{ Autovalores iniciales } & \multicolumn{3}{|c|}{$\begin{array}{l}\text { Sumas de extracción de cargas } \\
\text { al cuadrado }\end{array}$} & \multicolumn{3}{|c|}{$\begin{array}{l}\text { Sumas de rotación de cargas al } \\
\text { cuadrado }\end{array}$} \\
\hline & Total & $\begin{array}{c}\% \text { de } \\
\text { varianza }\end{array}$ & $\begin{array}{c}\% \\
\text { acumulado }\end{array}$ & Total & $\begin{array}{c}\% \text { de } \\
\text { varianza }\end{array}$ & $\begin{array}{c}\% \\
\text { acumulado }\end{array}$ & Total & $\begin{array}{c}\% \text { de } \\
\text { varianza }\end{array}$ & $\begin{array}{c}\% \\
\text { acumulado }\end{array}$ \\
\hline 1 & 2.724 & 34.045 & 34.045 & 2.724 & 34.045 & 34.045 & 2.458 & 30.719 & 30.719 \\
\hline 2 & 1.325 & 16.567 & 50.612 & 1.325 & 16.567 & 50.612 & 1.591 & 19.893 & 50.612 \\
\hline 3 & .987 & 12.337 & 62.949 & & & & & & \\
\hline 4 & .894 & 11.181 & 74.130 & & & & & & \\
\hline 5 & .801 & 10.012 & 84.142 & & & & & & \\
\hline 6 & .478 & 5.971 & 90.113 & & & & & & \\
\hline 7 & .449 & 5.618 & 95.731 & & & & & & \\
\hline 8 & .342 & 4.269 & 100.000 & & & & & & \\
\hline
\end{tabular}

Fuente: Elaboración propia. 
ORIENTACIÓN A OBJETIVOS DE LOGRO EN ESTUDIANTES DE EDUCACIÓN SUPERIOR.

En cuanto a los autovalores de la varianza total vistos en la tabla 4, los cuales expresan la cantidad por cada factor; los porcentajes de varianza explicada asociados a cada factor, se obtienen dividiendo su correspondiente autovalor por la suma de los autovalores. Por defecto, se extraen tantos factores como autovalores mayores que 1 tiene la matriz analizada. En este caso, hay 2 autovalores mayores que 1, uno con el valor de 2.458 y el otro con valor de 1.591, esos 2 valores logran explicar en un $50.61 \%$ de la varianza de los datos originales. También, se muestran para cada factor con un autovalor a 1, la suma de las saturaciones al cuadrado.

\section{Análisis de fiabilidad}

Para realizar la prueba de fiabilidad, se utilizó el coeficiente Alfa de Cronbach, porque es una de las medidas más recomendadas a utilizar para medir la correlación de los ítems de un instrumento de encuesta (Oviedo, 2005); además que este coeficiente puede ayudar a detectar como mejorar el instrumento excluyendo ítems y viceversa; es decir, cuanto se disminuye la fiabilidad del instrumento al dejar los ítems inadecuados (Ledesma, Molina, \& Valero, 2002). En cuanto a este análisis se obtuvo un resultado de .70 (Tabla 5), el cual quiere decir que tiene un nivel aceptable en cuanto a la integración de los ítems.

Tabla 5

Estadísticas de fiabilidad

\begin{tabular}{ll}
\hline Alfa de Cronbach & No. de elementos \\
\hline .70 & 8 \\
\hline
\end{tabular}

Fuente: Elaboración propia.

\section{Prueba T}

Se realizó una prueba T para analizar si existe diferencia entre género (hombres vs mujeres).

Tabla 6

Estadísticas de grupo

\begin{tabular}{lccccc}
\hline \multirow{3}{*}{ Total de la escala } & Género & $\mathrm{N}$ & Media & Desviación estándar & Media de error estándar \\
\cline { 2 - 6 } & Mujeres & 56 & 33.2143 & 3.88336 & .51894 \\
& Hombres & 48 & 32.4375 & 4.21197 & .60795 \\
\hline
\end{tabular}

Fuente: Elaboración propia.

De acuerdo con los resultados de la tabla 6 , se puede deducir que las medias son ligeramente similares con un 33.2143 para mujeres y un 32.4375 para hombres, habiendo una diferencia de 0.77 . 
Tabla 7

Prueba de muestras independientes

\begin{tabular}{|c|c|c|c|c|c|c|c|c|c|c|}
\hline \multirow{5}{*}{$\begin{array}{l}\text { Total } \\
\text { de } \\
\text { escala }\end{array}$} & \multirow[b]{4}{*}{$\begin{array}{l}\text { Se asumen } \\
\text { varianzas } \\
\text { iguales }\end{array}$} & \multicolumn{2}{|c|}{$\begin{array}{l}\text { Prueba de Levene } \\
\text { de calidad de } \\
\text { varianzas }\end{array}$} & \multicolumn{7}{|c|}{ Prueba t para la igualdad de medias } \\
\hline & & \multirow[t]{2}{*}{$\mathrm{F}$} & \multirow[t]{2}{*}{ Sig. } & \multirow[t]{2}{*}{$\mathrm{t}$} & \multirow[t]{2}{*}{ gl } & \multirow{2}{*}{$\begin{array}{c}\text { Sig. } \\
\text { (bilateral) }\end{array}$} & \multirow{2}{*}{$\begin{array}{l}\text { Diferencia } \\
\text { de } \\
\text { medias }\end{array}$} & \multirow{2}{*}{$\begin{array}{l}\text { Diferencia } \\
\text { de error } \\
\text { estándar }\end{array}$} & \multicolumn{2}{|c|}{$\begin{array}{l}95 \% \text { de intervalo de } \\
\text { confianza de la } \\
\text { diferencia }\end{array}$} \\
\hline & & & & & & & & & Inferior & Superior \\
\hline & & .101 & .751 & .978 & 102 & .330 & .77679 & .79429 & -.79869 & 2.35226 \\
\hline & $\begin{array}{l}\text { No se asumen } \\
\text { varianzas } \\
\text { iguales }\end{array}$ & & & .972 & 96.612 & .334 & .77679 & .79931 & -.80970 & 2.36327 \\
\hline
\end{tabular}

Fuente: Elaboración propia.

Analizando los resultados de la significancia bilateral de la tabla 7 (valor p) el cual es $0.330>$ 0.05 , se concluye que no hay una diferencia significativa entre los resultados de orientación a objetivos académicos de hombres vs mujeres. Se puede dar por hecho, que las medias son iguales entre ambos resultados, dicho de otra manera, la media del total de la escala en cuanto a la orientación de objetivos académicos es igual entre hombres y mujeres.

\section{Prueba F}

Se realizó la prueba F, comparando los resultados de los semestres de los encuestados, los cuales son de los semestres de segundo-doceavo (se tomó como un conjunto 10, 11 y 12, debido a que son de semestres rezagados).

Tabla 8

Pruebas de normalidad

\begin{tabular}{|c|c|c|c|c|c|c|c|}
\hline & \multirow[b]{2}{*}{ Semestre } & \multicolumn{3}{|c|}{ Kolmogorov-Smirnov ${ }^{\mathrm{a}}$} & \multicolumn{3}{|c|}{ Shapiro-Wilk } \\
\hline & & Estadístico & $\mathrm{gl}$ & Sig. & Estadístico & gl & Sig. \\
\hline \multirow[t]{10}{*}{ Total de la escala } & 2 do & .238 & 10 & .116 & .872 & 10 & .104 \\
\hline & 3ro & .149 & 26 & .140 & .960 & 26 & .393 \\
\hline & 4to & .196 & 4 & . & .984 & 4 & .925 \\
\hline & 5to & .210 & 19 & .028 & .901 & 19 & .050 \\
\hline & 6 to & .183 & 6 & $.200^{\star}$ & .940 & 6 & .661 \\
\hline & $7 \mathrm{mo}$ & .233 & 7 & $.200^{\circ}$ & .929 & 7 & .545 \\
\hline & $8 \mathrm{vo}$ & .130 & 12 & .200 & .944 & 12 & .555 \\
\hline & 9 no & .184 & 12 & $.200^{*}$ & .914 & 12 & .244 \\
\hline & Rezagados & .260 & 2 & . & & & \\
\hline & Rezagados & .279 & 5 & .200 & .885 & 5 & .335 \\
\hline
\end{tabular}

Fuente: Elaboración propia. 


\section{Original}

ORIENTACIÓN A OBJETIVOS DE LOGRO EN ESTUDIANTES DE EDUCACIÓN SUPERIOR.

Analizando los resultados de la tabla 8, la prueba Shapiro-Wilk, se puede observar que los valores de significancia son mayores a 0.05 , lo que quiere decir que hay distribuciones normales. Para encontrar las diferencias se aplicará la prueba F de ANOVA.

Tabla 9

Estadístico ANOVA

\begin{tabular}{|c|c|c|c|c|c|}
\hline & $\begin{array}{c}\text { Suma de } \\
\text { cuadrados }\end{array}$ & gl & $\begin{array}{c}\text { Media } \\
\text { cuadrática }\end{array}$ & $\mathrm{F}$ & Sig. \\
\hline Entre grupos & 50.130 & 5 & 10.026 & .603 & .698 \\
\hline Dentro de grupos & 1628.706 & 98 & 16.619 & & \\
\hline Total & 1678.837 & 103 & & & \\
\hline
\end{tabular}

Fuente: Elaboración propia.

Tabla 10

Prueba Tukey comparaciones múltiples

\begin{tabular}{|c|c|c|c|c|c|c|}
\hline \multirow[b]{2}{*}{ (I) Carrera } & \multirow{2}{*}{\multicolumn{2}{|c|}{$\begin{array}{l}\text { Diferencia de } \\
\text { medias (I-J) }\end{array}$}} & \multirow[b]{2}{*}{ Error estándar } & \multicolumn{3}{|c|}{$95 \%$ de intervalo de confianza } \\
\hline & & & & Sig. & Límite inferior & Límite superior \\
\hline \multirow[t]{5}{*}{1.00} & 2.00 & -.85606 & .96524 & .949 & -3.6619 & 1.9498 \\
\hline & 3.00 & .29167 & 1.62216 & 1.000 & -4.4238 & 5.0071 \\
\hline & 4.00 & -.08333 & 2.16999 & 1.000 & -6.3912 & 6.2246 \\
\hline & 5.00 & -.83333 & 2.97720 & 1.000 & -9.4877 & 7.8210 \\
\hline & 6.00 & 1.10417 & 1.26202 & .952 & -2.5644 & 4.7727 \\
\hline \multirow[t]{5}{*}{2.00} & 1.00 & .85606 & .96524 & .949 & -1.9498 & 3.6619 \\
\hline & 3.00 & 1.14773 & 1.56689 & .977 & -3.4070 & 5.7025 \\
\hline & 4.00 & .77273 & 2.12899 & .999 & -5.4160 & 6.9614 \\
\hline & 5.00 & .02273 & 2.94745 & 1.000 & -8.5452 & 8.5906 \\
\hline & 6.00 & 1.96023 & 1.19014 & .570 & -1.4994 & 5.4198 \\
\hline \multirow[t]{5}{*}{3.00} & 1.00 & -.29167 & 1.62216 & 1.000 & -5.0071 & 4.4238 \\
\hline & 2.00 & -1.14773 & 1.56689 & .977 & -5.7025 & 3.4070 \\
\hline & 4.00 & -.37500 & 2.49646 & 1.000 & -7.6319 & 6.8819 \\
\hline & 5.00 & -1.12500 & 3.22291 & .999 & -10.4936 & 8.2436 \\
\hline & 6.00 & .81250 & 1.76526 & .997 & -4.3189 & 5.9439 \\
\hline \multirow[t]{5}{*}{4.00} & 1.00 & .08333 & 2.16999 & 1.000 & -6.2246 & 6.3912 \\
\hline & 2.00 & -.77273 & 2.12899 & .999 & -6.9614 & 5.4160 \\
\hline & 3.00 & .37500 & 2.49646 & 1.000 & -6.8819 & 7.6319 \\
\hline & 5.00 & -.75000 & 3.53052 & 1.000 & -11.0128 & 9.5128 \\
\hline & 6.00 & 1.18750 & 2.27894 & .995 & -5.4371 & 7.8121 \\
\hline \multirow[t]{5}{*}{5.00} & 1.00 & .83333 & 2.97720 & 1.000 & -7.8210 & 9.4877 \\
\hline & 2.00 & -.02273 & 2.94745 & 1.000 & -8.5906 & 8.5452 \\
\hline & 3.00 & 1.12500 & 3.22291 & .999 & -8.2436 & 10.4936 \\
\hline & 4.00 & .75000 & 3.53052 & 1.000 & -9.5128 & 11.0128 \\
\hline & 6.00 & 1.93750 & 3.05752 & .988 & -6.9504 & 10.8254 \\
\hline \multirow[t]{5}{*}{6.00} & 1.00 & -1.10417 & 1.26202 & .952 & -4.7727 & 2.5644 \\
\hline & 2.00 & -1.96023 & 1.19014 & .570 & -5.4198 & 1.4994 \\
\hline & 3.00 & -.81250 & 1.76526 & .997 & -5.9439 & 4.3189 \\
\hline & 4.00 & -1.18750 & 2.27894 & .995 & -7.8121 & 5.4371 \\
\hline & 5.00 & -1.93750 & 3.05752 & .988 & -10.8254 & 6.9504 \\
\hline
\end{tabular}

Fuente: Elaboración propia. 
ORIENTACIÓN A OBJETIVOS DE LOGRO EN ESTUDIANTES DE EDUCACIÓN SUPERIOR.

De acuerdo con los resultados de la tabla 9, el cual tuvo un valor de .603, se puede concluir que el estadístico $\mathrm{F}$ no es significativo. Además, se realizó un análisis de comparaciones múltiples en la tabla 10, en las cuales se observó que no existe diferencia significativa entre ninguno de los grupos de estudio.

\section{CONCLUSIONES}

Después de aplicar las diferentes pruebas al cuestionario, para cumplir con el objetivo de analizar si es un instrumento fiable que ayude a determinar cuál es la orientación a objetivos académicos de los estudiantes de nivel superior, se puede deducir que:

- De acuerdo con el análisis factorial, la variable 1: La opinión que los demás tienen sobre lo bien que puedo trabajar son importantes para mí, es la variable peor explicada, pues del modelo propuesto solo puede explicar en un $22.5 \%$ de la variable original (orientación a objetivos académicos) por lo tanto, ésta variable puede ser suprimida del cuestionario porque no aporta ningún valor para determinar las orientaciones a objetivos.

- El nivel del coeficiente Alfa de Cronbach es igual a 0.70 lo cual indica que el cuestionario aplicado tiene un nivel aceptable de fiabilidad.

- De acuerdo con la prueba T, se deduce que no existe diferencia significativa entre la orientación a objetivos académicos de logro de mujeres respecto de hombres

- Además, se comprobó que no existe diferencia significativa entre los resultados por semestres de los alumnos, los cuales pertenecen a los semestres 2-12.

Para finalizar, el cuestionario propuesto es fiable para medir la orientación a objetivos académicos en estudiantes de nivel superior; sin embargo, se recomienda eliminar la primera variable (Tabla 1), porque explica en un nivel muy bajo la orientación a objetivos de logro del modelo propuesto.

Además, se encontró que no hay diferencia significativa en la orientación de objetivos académicos de hombres respecto de mujeres, comparando con la teoría del estudio en la cual mencionan que existe una diferencia muy marcada, porque los hombres tienden a orientarse hacia objetivos de rendimiento y las mujeres a objetivos de aprendizaje, esto quiere decir, que no hay una tendencia marcada en estudiantes (hombres y mujeres) en orientación a objetivos académicos de los estudiantes encuestados del Instituto Tecnológico de Celaya. 
ORIENTACIÓN A OBJETIVOS DE LOGRO EN ESTUDIANTES DE EDUCACIÓN SUPERIOR.

\section{REFERENCIAS BIBLIOGRÁFICAS}

Ames, C. \&. Archer J. (1988). Achievement goals in the classroom: Students' learning strategies and motivation processes. Journal of Educational Psychology, 80(3), 260-267.

Ames, C. (1984). Achievement attributions and self-instructions under competitive and individualistic goal structures. Journal of Educational Psychology, 76(3), 478-487.

Ames, C. (1992). Classrooms: Goals, structures, and student motivation. Journal of Educational Psychology, 84(3), 261-271.

Atkinson, J. W. (1957). Motivational determinants of risk-taking behavior. Psychological Review, 64(6, Pt. 1), 359-372.

Blackwell, L. S., Trzesniewski, K. H. \& Dweck, C. S. (2007). Implicit Theories of Intelligence Predict Achievement Across an Adolescent Transition: A Longitudinal Study and an Intervention. Child Development, 78(1), 246-263.

Button, S. B., Mathieu, J. E. \& Zajac, D. M., (1996). Goal orientation in organizational research: A conceptual and empirical foundation. Organizational behavior and human decision processes, 67(1), 26-48.

Cimpian, A., Arce, H. M. C. \& Markman, E. M. \& Dweck, C. S. (2007). Subtle Linguistic Cues Affect Children's Motivation. Psychological Science, 18(4), 314-316.

De la Fuente, S. (2011). Análisis factorial.Facultad de Ciencias Económicas y Empresariales, Universidad Autónoma de Madrid, Madrid, España.

Diener, C. I. \& Dweck, C. S. (1978). An analysis of learned helplessness: Continuous changes in performance, strategy, and achievement cognitions following failure. Journal of Personality and Social Psychology, 36(5), 451-462.

Dweck, C. S. (1986). Motivational processes affecting learning. American Psychologist, 4(10), 1040-1048.

Dweck, C. S. \& Leggett, E. L. (1988). A social cognitive approach to motivation and personality. Psychological Review, 95(2), 256-273.

Dweck, C. S. (2010). Even geniuses work hard. Educational Leadership, 68(1), 16-20.

Eccles, J. S., Midgley, C., Wigfield, A., Buchanan, C. M., Reuman, D. \& Flanagan, C. (1993). The impact of stage-environment fit on young adolescents experiences in schools and in families. American Psychologist, 48(2), 90-101.

Elliot, A. J. \& Harackiewicz, J. M. (1996). Approach and avoidance achievement goals and intrinsic motivation: A mediational analysis. Journal of Personality and Social Psychology, 70(3), 461-475.

Hattie, J. \& Timperley, H. (2007). The Power of Feedback. Review of Educational Research, 77 (1), 81-112. 
ORIENTACIÓN A OBJETIVOS DE LOGRO EN ESTUDIANTES DE EDUCACIÓN SUPERIOR.

Henderlong, J. \&. Lepper, M. R. (2002). The effects of praise on children's intrinsic motivation: A review and synthesis. Psychological Bulletin, 128(5), 774-795.

Kamins, M. L. \& Dweck, C. S. (1999). Person versus process praise and criticism: Implications for contingent self-worth and coping. Developmental Psychology, 35(3), 835-847.

Ledesma, R., Molina, G. \& Valero, P. (2002). Análisis de consistencia interna mediante Alfa de Cronbach: un programa basado en gráficos dinámicos. Psico-USF, 7(2), 143-152.

Meece, J. L. \& Holt, K. (1993). A pattern analysis of students' achievement goals. Journal of Educational Psychology, 85(4), 582-590.

Midgley, C. \& Anderman, E. \&. Hicks, L. (1995). Differences between Elementary and Middle School Teachers and Students: A Goal Theory Approach. The Journal of Early Adolescence, 15(1), 90-113.

Mueller, C. M. \& Dweck, C. S. (1998). Praise for intelligence can undermine children's motivation and performance. Journal of Personality and Social Psychology, 75(1), 33-52.

Oviedo, H. C. \& Arias A. C. (2005). Aproximación al uso del coeficiente alfa de Cronbach. Revista colombiana de psiquiatría, 34(4), 572-580.

Senko, C. \& Harackiewicz, J. M. (2002). Performance goals: The moderating roles of context and achievement orientation. Journal of Experimental Social Psychology, 38(6), 603-610.

Shatz, I. (2015). The negative impact of goal-oriented instructions. Educational Studies, 41(5), 476-480.

Weiner, B. (1979). A theory of motivation for some classroom experiences. Journal of Educational Psychology, 71(1), 3-25.

Wolters, C. A. (2004). Advancing Achievement Goal Theory: Using Goal Structures and Goal Orientations to Predict Students' Motivation, Cognition, and Achievement. Journal of Educational Psychology, 96(2), 236-250. 